\title{
Prediction of TERTp-mutation status in IDH-wildtype high-grade gliomas using pre-treatment dynamic $\left[{ }^{18} \mathrm{~F}\right] \mathrm{FET}$ PET radiomics
}

\author{
Zhicong $\mathrm{Li}^{1}$ • Lena Kaiser ${ }^{1}$. Adrien Holzgreve ${ }^{1} \cdot$ Viktoria C. Ruf $^{2} \cdot$ Bogdana Suchorska $^{3,4} \cdot$ Vera Wenter $^{1}$. \\ Stefanie Quach ${ }^{3}$. Jochen Herms ${ }^{2}$. Peter Bartenstein ${ }^{1,5}$. Jörg-Christian Tonn ${ }^{3,5}$. Marcus Unterrainer ${ }^{6}$. \\ Nathalie L. Albert ${ }^{1,5}$
}

Received: 10 June 2021 / Accepted: 5 August 2021 / Published online: 7 September 2021

C The Author(s) 2021, corrected publication 2022

\begin{abstract}
Purpose To evaluate radiomic features extracted from standard static images (20-40 min p.i.), early summation images (5-15 min p.i.), and dynamic $\left[{ }^{18} \mathrm{~F}\right] \mathrm{FET}$ PET images for the prediction of TERTp-mutation status in patients with IDHwildtype high-grade glioma.

Methods A total of 159 patients (median age 60.2 years, range 19-82 years) with newly diagnosed IDH-wildtype diffuse astrocytic glioma (WHO grade III or IV) and dynamic $\left[{ }^{18} \mathrm{~F}\right] \mathrm{FET}$ PET prior to surgical intervention were enrolled and divided into a training $(n=112)$ and a testing cohort $(n=47)$ randomly. First-order, shape, and texture radiomic features were extracted from standard static (20-40 min summation images; $\mathrm{TBR}_{20-40}$ ), early static (5-15 min summation images; $\mathrm{TBR}_{5-15}$ ), and dynamic (time-to-peak; TTP) images, respectively. Recursive feature elimination was used for feature selection by 10 -fold cross-validation in the training cohort after normalization, and logistic regression models were generated using the radiomic features extracted from each image to differentiate TERTp-mutation status. The areas under the ROC curve (AUC), accuracy, sensitivity, specificity, and positive and negative predictive value were calculated to illustrate diagnostic power in both the training and testing cohort.

Results The TTP model comprised nine selected features and achieved highest predictability of TERTp-mutation with an AUC of 0.82 (95\% confidence interval $0.71-0.92$ ) and sensitivity of $92.1 \%$ in the independent testing cohort. Weak predictive capability was obtained in the $\mathrm{TBR}_{5-15}$ model, with an AUC of 0.61 (95\% CI 0.42-0.80) in the testing cohort, while no predictive power was observed in the $\mathrm{TBR}_{20-40}$ model.

Conclusions Radiomics based on TTP images extracted from dynamic $\left[{ }^{18}\right.$ F]FET PET can predict the TERTp-mutation status of IDH-wildtype diffuse astrocytic high-grade gliomas with high accuracy preoperatively.
\end{abstract}

Keywords Radiomics $\cdot\left[{ }^{18}\right.$ F $]$ FET PET $\cdot$ TERTp-mutation $\cdot$ Glioma

This article is part of the Topical Collection on Oncology - Brain

Nathalie L. Albert

nathalie.albert@med.uni-muenchen.de

1 Department of Nuclear Medicine, University Hospital, LMU Munich, Marchioninistr. 15, 81377 Munich, Germany

2 Center for Neuropathology and Prion Research, LMU Munich, Munich, Germany

3 Department of Neurosurgery, University Hospital, LMU Munich, Munich, Germany
4 Department of Neurosurgery, Sana Hospital, Duisburg, Germany

5 German Cancer Consortium (DKTK), Partner Site Munich, German Cancer Research Center (DKFZ), Heidelberg, Germany

6 Department of Radiology, University Hospital, LMU Munich, Munich, Germany 


\section{Introduction}

Mutations in the telomerase reverse transcriptase promoter (TERTp), leading to telomerase activation and lengthened telomeres, play an important role in the formation of brain cancer and individual prognosis [1-3]. In diffuse astrocytic high-grade gliomas without mutation of the isocitrate dehydrogenase gene (IDH-wildtype), TERTp mutations are reported to be associated with poor overall survival [4-6]. Molecular genetic analysis of the TERTp-mutation status has therefore gained increasing attention in the clinical routine diagnosis of IDH-wildtype diffuse astrocytic gliomas and will be included in the upcoming glioma WHO classification [7-9].

Molecular imaging using positron emission tomography (PET) with radiolabelled amino acids such as $O-\left(2-\left[{ }^{18} \mathrm{~F}\right]-\right.$ fluoroethyl)-L-tyrosine ( $\left.\left[{ }^{18} \mathrm{~F}\right] \mathrm{FET}\right)$ is a useful tool for the characterization and evaluation of primary brain neoplasms [10-12], and its application in the clinical management of brain tumour patients has been recommended by the Response Assessment in Neuro-Oncology (RANO) Working Group [13-17]. While static image data (standard 20-40 min summation images) are particularly used for the delineation of the tumour extent, the assessment of dynamic $\left[{ }^{18} \mathrm{~F}\right] \mathrm{FET}$ PET data has been shown to provide additional information about tumour biology [18]. More aggressive gliomas (i.e. high-grade gliomas and/or IDH-wildtype gliomas) were shown to be characterized by a high tracer uptake within the first 5-15 min post injection (p.i.) with subsequent curve decrease, while less aggressive gliomas (i.e. low grade gliomas and/or IDH-mutant gliomas) typically show a slowly increasing $\left[{ }^{18} \mathrm{~F}\right] \mathrm{FET}$ uptake with highest values in the later time frames $[12,19,20]$. As the early peak uptake in aggressive gliomas is missed in the standard 20-40 min p.i. summation images, it does not surprise that the maximal tumour-to-background ratio $\left(\mathrm{TBR}_{\max }\right)$ evaluation obtained in early summation images (5-15 min p.i.) was reported to perform better than the standard static $\mathrm{TBR}_{\max }$ values (20-40 min p.i.) for the differentiation between low-grade and high-grade gliomas [17], which led to the suggestion to include these early summation images for a better glioma characterization. Another interesting parameter derived from dynamic $\left[{ }^{18} \mathrm{~F}\right]$ FET PET is the minimal time-to-peak $\left(\mathrm{TTP}_{\min }\right)$, which is extracted from the time-activity-curves and was reported to provide prognostic information [21]. Interestingly, an early $\mathrm{TTP}_{\text {min }}$ was associated with an aggressive disease course in newly diagnosed gliomas and was able to predict an IDH-wildtype status [22, 23]. Yet, in our recently published study investigating $\left[{ }^{18} \mathrm{~F}\right] \mathrm{FET}$ uptake characteristics in TERTp mutant and TERTp wildtype glioblastomas, neither the standard $\mathrm{TBR}_{\max }$ as static parameter nor $\mathrm{TTP}_{\text {min }}$ as dynamic parameter were associated with the TERTp-mutation status [24].
In recent years, radiomics have been increasingly investigated as a promising non-invasive tool for accurate diagnosis and prognosis assessment by converting medical images into high-dimensional quantitative image features and establishing predictive models [25-32]. However, radiomics have not been applied for the detection of TERTp mutations on $\left[{ }^{18} \mathrm{~F}\right]$ FET PET images so far. Therefore, the aim of this study was to evaluate radiomic features extracted from standard static images (20-40 min p.i.), early summation images (5-15 min p.i.) as well as dynamic $\left[{ }^{18} \mathrm{~F}\right] \mathrm{FET}$ PET images for the prediction of the TERTp-mutation status in patients with newly diagnosed IDH-wildtype diffuse astrocytic highgrade glioma.

\section{Materials and methods}

\section{Patients}

Patients with primary diagnosis of a glioma who had received a pre-treatment dynamic $\left[{ }^{18} \mathrm{~F}\right]$ FET PET scan at the Department of Nuclear Medicine of the LMU Munich between December 2005 and June 2016 were screened for this retrospective study. Inclusion criteria were (1) neuropathologically confirmed IDH-wildtype diffuse astrocytic gliomas (WHO grade III or IV) according to the updated 2016 WHO classification [33], (2) availability of the TERTpmutation status, and (3) pre-treatment dynamic $\left[{ }^{18} \mathrm{~F}\right] \mathrm{FET}$ PET scan (ECAT EXACT HR +, Siemens Healthineers, Inc., Erlangen, Germany Siemens Medical Systems, Inc., Erlangen, Germany). [ $\left.{ }^{18} \mathrm{~F}\right] \mathrm{FET}$-negative gliomas (tumourto-background ratio, TBR $<1.6$ ) were excluded. All patients had given written informed consent prior to the PET scan as part of the clinical routine. The retrospective analysis of PET imaging data was approved by the institutional ethics committee (604-16). A total of $61 \%$ of the investigated patients (97/159) have been evaluated in a previous study [24].

\section{Histopathology and molecular genetic analysis}

Histopathology and molecular genetic analyses were performed at the Institute of Neuropathology, LMU Munich, Germany. All patients initially classified according to the 2007 WHO brain tumour classification [34] were reclassified according to the 2016 WHO classification [33]. The IDH-mutation status and TERTp-mutation status were evaluated according to clinical standard protocols $[35,36]$.

\section{$\left[{ }^{18}\right.$ F]FET PET imaging}

$\left[{ }^{18} \mathrm{~F}\right]$ FET PET scans were performed at the Department of Nuclear Medicine, LMU Munich, Germany. Images were acquired by using an ECAT EXACT HR + PET scanner 
(Siemens Healthineers, Inc., Erlangen, Germany) with the standard protocol $[11,37]$. Exactly $180 \mathrm{MBq}$ of $\left[{ }^{18} \mathrm{~F}\right] \mathrm{FET}$ were injected after a 15 -min transmission scan with a ${ }^{68} \mathrm{Ge}$ rotating rod source. After tracer injection up to $40 \mathrm{~min}$ post injection in 3 -D mode consisting of 16 frames $(7 \times 10 \mathrm{~s}$, $3 \times 30 \mathrm{~s}, 1 \times 2 \mathrm{~min}, 3 \times 5 \mathrm{~min}$, and $2 \times 10 \mathrm{~min}$ ) with a reconstructed voxel size of $2.03 \times 2.03 \times 2.43 \mathrm{~mm}^{3}$ and matrix size of $128 \times 128 \times 63$, dynamic emission recording was finished. Two-dimensional filtered back-projection reconstruction algorithm using a 4.9-mm Hann Filter was applied for image reconstruction, then corrected for attenuation, decay, dead time, and random and scattered coincidences. When relevant motion was visible in dynamic PET data, a frame-wise correction was performed by using PMOD fusion tool (version 3.5, PMOD Technologies, Zurich, Switzerland) after framewise checking for motion.

\section{Segmentation of tumour volumes and brain background}

First, a background activity was extracted from a large crescent-shaped volume of interest (VOI) in the contralateral healthy hemisphere as published previously [38]. For tumour segmentation, a VOI was drawn using a TBR-threshold of 1.6 in static $20-40$ min p.i. summation images as suggested by Pauleit et al. [39]. All segmentations were processed within the PMOD View tool (version 3.5, PMOD Technologies, Zurich, Switzerland).

\section{Image normalization and TTP image generation}

We used the in-house developed software described previously by Kaiser et al. [40] (C++ with integration of the ROOT data analysis framework, version 6.22/08, Cern, Switzerland and ITK segmentation and registration toolkit 4.13.3, National Library of Medicine) to generate voxel-wise parametric images. Then we normalized the image values with the mean background value derived from each image by using the VOI of background to generate early 5-15 min p.i. $\left(\mathrm{TBR}_{5-15}\right)$ and late $20-40 \mathrm{~min}$ p.i. $\left(\mathrm{TBR}_{20-40}\right) \mathrm{TBR}$ images. For TTP images, time-activity curves (TAC) were extracted from each voxel, which were then classified according to the time frame reaching the peak uptake (i.e. (1) $<5 \mathrm{~min}$, (2) 5-10 min, (3) 10-15 min, (4) 15-20 min, (5) 20-30 min, and (6) 30-40 min). To avoid influence from early blood flush, TTP analyses did not include the first $2.7 \mathrm{~min}$ p.i. [40]. In case of a positive late slope (15-40 min p.i.), the TTP was always assigned to group 6 .

\section{Radiomic feature extraction}

Radiomic features from parametric images were extracted with PyRadiomics (version 3.0.1) [41] as introduced previously by Kaiser et al. [42], and complied with the Imaging Biomarker Standardization Initiative (IBSI) guidelines [43]. Before extraction, images were resampled to isotropic voxels using linear interpolation in PyRadiomics (size $2.03 \times 2.03 \times 2.03 \mathrm{~mm}^{3}$ ). Classes of features extracted from $\mathrm{TBR}_{5-15}, \mathrm{TBR}_{20-40}$, and TTP images included first-order features, shape features, and texture features. No image filters were used. The chosen fixed intensity bin size was set to the average interquartile range divided by 4 , which led to 0.18 for $\mathrm{TBR}_{5-15}$ images and 0.13 for $\mathrm{TBR}_{20-40}$ images $[42,44]$. As the smallest time frame duration considered in the TTP categories was $5 \mathrm{~min}$, this was used as the fixed bin width for radiomics calculation of TTP images.

\section{Feature selection}

Before feature extraction, a stratified random split was used to assign $70 \%$ of the patients to the training cohort $(n=112)$ and the remaining $30 \%$ to the testing cohort $(n=47)$, with a balanced distribution of TERTp-wildtype and TERTp-mutation.

Features were standardized as follows: for each feature, we calculated the mean value and the standard deviation. The mean value was subtracted from each individual value, which was then divided by the standard deviation. Feature normalization was computed only in the training cohort and then applied on the testing cohort. Since the number of features was large, we compared the similarity of each feature pair. If the Pearson correlation coefficient (PCC) value of the feature pair was larger than 0.99 , we removed one of them. After this process, the number of the features was reduced and each feature was independent to each other. The recursive feature elimination (RFE) based on logistic regression classifier was performed to reduce redundant features and select potential TERTp-mutation related features [45]. Considering the imbalance of comparison groups, we performed the weighted logistic regression in the 'balanced' mode, which gives higher weight to the minority class and lower weight to the majority class and therefore automatically adjusts weights inversely proportional to class frequencies in the input data [46]. Each iteration removes a feature which is considered least important. After stratified splitbased 10-fold cross-validation, the area under the receiver operating characteristic curve (AUC) of the model in the training cohort was used to determine the optimal number of features.

\section{Model construction and testing}

Logistic regression (LR) models were built to predict the TERTp-mutation status by fitting the selected radiomic features. Each model was generated by using only the radiomic 
features extracted from each image (i.e. $\mathrm{TBR}_{5-15}, \mathrm{TBR}_{20-40}$, and TTP images) separately. According to the coefficients of selected features generated by the LR models [47], the risk probability of TERTp-mutation was calculated by the following formula:

$\mathrm{P}(y=1 \mid x ; \theta)=\frac{1}{1+e^{-\theta^{\mathrm{T}} x}}$

$x$ is the value of selected features, $\theta$ is the coefficient of selected features, and $\theta_{0}$ represents the intercept. In case of $\mathrm{P}>0.5$, TERTp-mutation status was considered as positive by the LR model.

Model testing was applied to the independent testing cohort, which was not involved in the process of model training. The workflow of the process is presented in Fig. 1.

\section{Statistical analysis}

To evaluate the model performance, receiver operating characteristic curve (ROC) analysis was performed in the training and testing cohort. The AUC was calculated as quantitative measure to illustrate diagnostic power. The accuracy, sensitivity, specificity, positive predictive value (PPV), and negative predictive value (NPV) were calculated. 95\% confidence intervals (CI) were calculated by using a non-parametric bootstrap method, which was repeated 1000 times to get a bootstrap distribution of the results.

Categorical variables or continuous variables were reported as numbers and percentages or as mean and standard deviation. Categorical variables were compared by the $\chi^{2}$ test, and continuous variables were compared by the Mann-Whitney $U$ test. $P<0.05$ were considered statistically significant. Statistical analyses were programmed in Python (v. 3.8.5; https://www.python.org/).

\section{Results}

\section{Patient characteristics}

A total of 159 patients (median age, 60.2 years; range, 19-82 years) were enrolled in this study. Exactly 31 patients (19.50\%) were diagnosed with TERTp-wildtype, and 128 patients had TERTp mutation. The clinical characteristics are presented in Table 1 . There were no significant differences between the training and testing cohorts with regard to age, sex, WHO grade, and TERTp mutation status, with TERTp-wildtype rates of $19.64 \%$ and $19.15 \%$, respectively.

\section{Radiomic feature extraction and selection}

In this study, 107 radiomic features of candidates were generated from standard static images (20-40 min p.i.), early summation images (5-15 min p.i.), and dynamic $\left[{ }^{18} \mathrm{~F}\right] \mathrm{FET}$ PET images respectively, including first-order statistics, shape-based features, and texture features. After PCC process, $80 \mathrm{TBR}_{20-40}$ features, $83 \mathrm{TBR}_{5-15}$ features, and $91 \mathrm{TTP}$ features were retained. For the $\mathrm{TBR}_{20-40}$ model, based on the AUC of the 10-fold cross-validation on the training cohort,

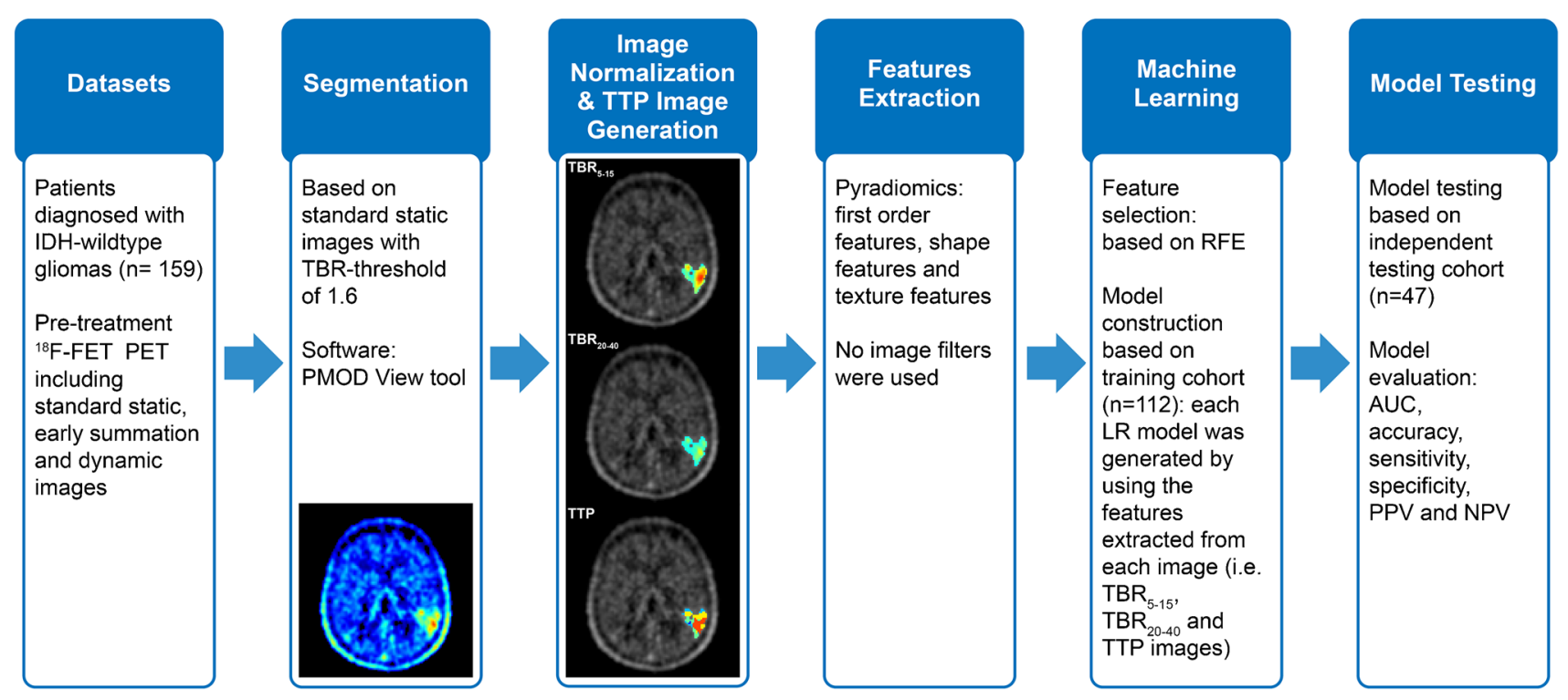

Fig. 1 The workflow of process. TBR tumour-to-background ratio, TTP time-to-peak, RFE recursive feature elimination, LR logistic regression, AUC area under the receiver operating characteristic curve, PPV positive predictive value, NPV negative predictive value 
Table 1 Clinical characteristics of the patients

\begin{tabular}{|c|c|c|c|c|c|}
\hline & \multicolumn{2}{|c|}{ Training cohort $(n=112)$} & \multicolumn{2}{|c|}{ Testing cohort $(n=47)$} & \multirow[b]{2}{*}{$P$} \\
\hline & TERTp-mutation & $\overline{\text { TERTp-wildtype }}$ & TERTp-mutation & TERTp-wildtype & \\
\hline Characteristic & $(n=90)$ & $(n=22)$ & $(n=38)$ & $(n=9)$ & 0.8958 \\
\hline Age, years & $58.1 \pm 12.3$ & & $59.2 \pm 11.2$ & & 0.3699 \\
\hline \multicolumn{6}{|l|}{ Sex } \\
\hline Female & $45(40.2 \%)$ & & $17(54.8 \%)$ & & 0.1449 \\
\hline Male & $67(59.8 \%)$ & & $14(45.2 \%)$ & & \\
\hline \multicolumn{6}{|l|}{ WHO grade } \\
\hline III & $39(34.8 \%)$ & & $14(29.8 \%)$ & & 0.5389 \\
\hline IV & $73(65.2 \%)$ & & $33(70.2 \%)$ & & \\
\hline
\end{tabular}

Data are means \pm standard deviations or numbers of patients with percentages in parentheses. $P$ value was derived from the univariate association analyses between each clinical parameter. Calculated by using the independent sample $t$ test for continuous variables and the $\chi^{2}$ test for categoric variables
14 features were finally selected to fit the LR model after performing the RFE method. For the $\mathrm{TBR}_{5-15}$ model and

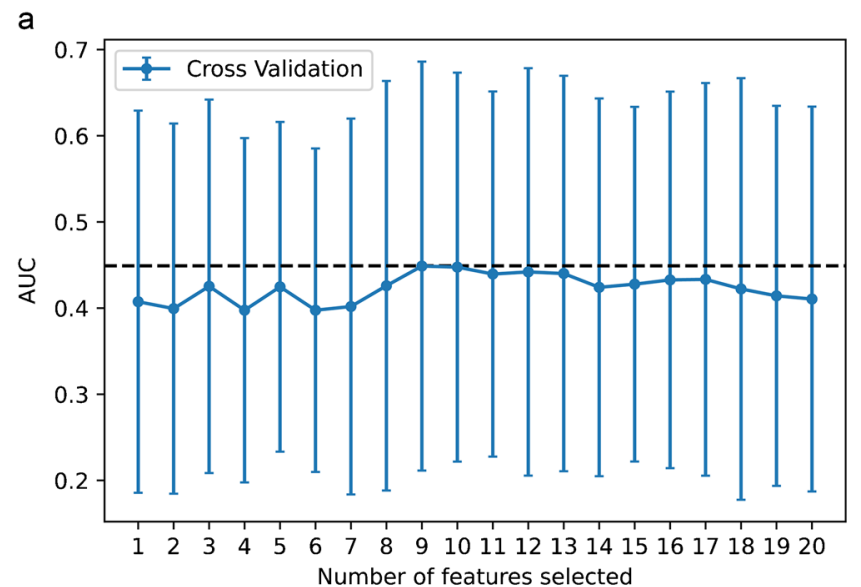

C

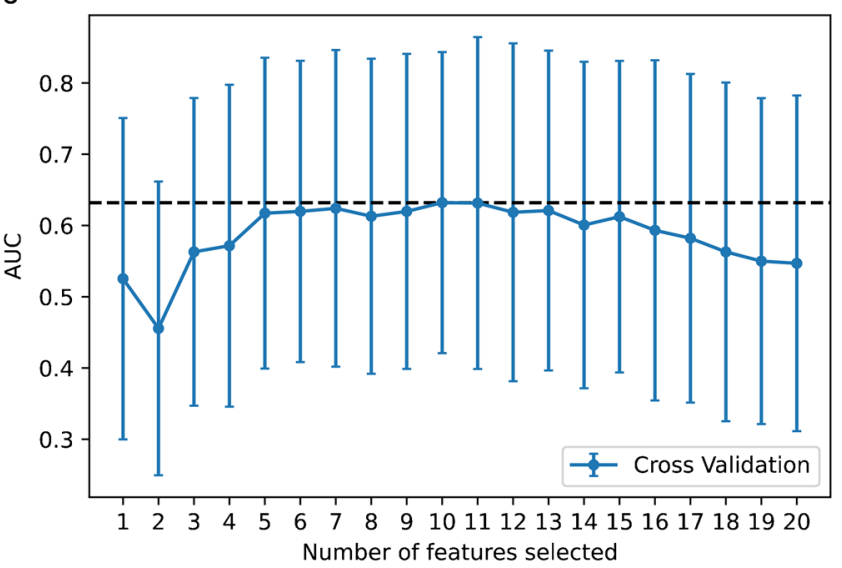

Fig. 2 The feature selection process of the RFE method. Each iteration removes a feature that is considered least important and corresponds to a 10 -fold cross-validation. After 10 -fold cross-validation, the AUC of the model in the training cohort was used to determine the optimal number of features. The minimum AUC of feature num- the TTP model, 9 features and 10 features were selected for inclusion in the LR model, respectively (Fig. 2).

b

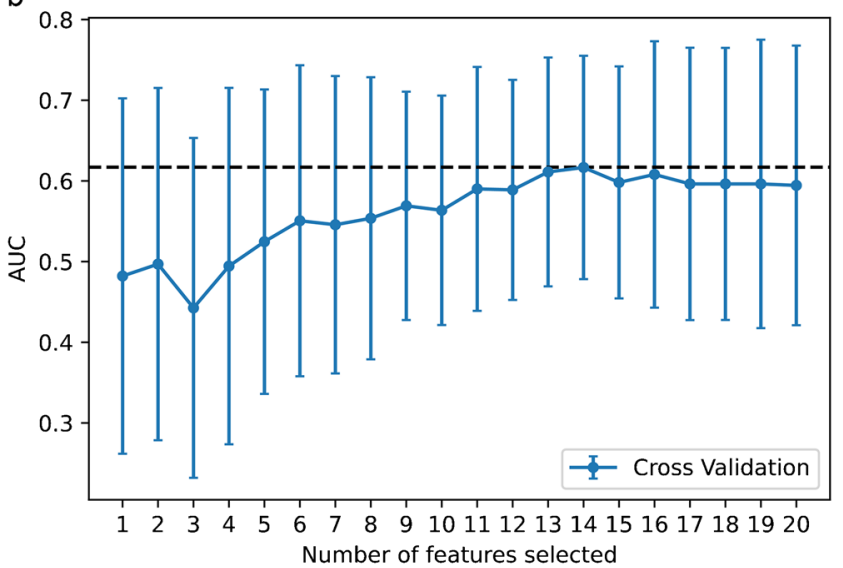

ber was selected. a $\mathrm{TBR}_{5-15}$ model, b $\mathrm{TBR}_{20-40}$, and c TTP model; 9,14 , and 10 features were selected respectively. RFE recursive feature elimination, AUC area under the receiver operating characteristic curve 


\section{Diagnostic Validation of the $\mathrm{TBR}_{20-40}$ model, $\mathrm{TBR}_{5-15}$ model, and TTP model}

According to the above-mentioned formula, the risk probabilities of TERTp-mutation were calculated. The coefficients of selected features in the $\mathrm{TBR}_{20-40}$ model and $\mathrm{TBR}_{5-15}$ model are shown in Table $\mathrm{S} 1$. The coefficients of selected features in the TTP model are shown in Table 2.

No predictive power was observed in the $\mathrm{TBR}_{20-40}$ model with an AUC of only 0.49 (95\% CI $0.30-0.69)$ in the testing cohort (AUC of 0.90 in the training cohort (95\% CI 0.85-0.95); see Fig. S1). The $\mathrm{TBR}_{5-15}$ model demonstrated weak predictive capability to predict a TERTp-mutation (Fig. 3a, b), with an AUC of 0.61 (95\% CI 0.42-0.80) in the testing cohort and an AUC of 0.80 (95\% CI 0.71-0.89) in the training cohort. The TTP model showed the strongest predictive power and achieved an AUC of 0.82 (95\% CI 0.71-0.92) and 0.90 (95\% CI 0.84-0.95) in the testing cohort and training cohort, respectively (Fig. 3c, d).

Detailed information about the performance of each model is shown in Table 3 .

\section{Discussion}

Our study showed that radiomics based on dynamic $\left[{ }^{18} \mathrm{~F}\right]$ FET PET data can reliably predict the TERTp-mutation status of IDH-wildtype diffuse astrocytic high-grade gliomas. Best predictability was reached using the TTP model derived from dynamic PET, and weak predictive capability was obtained with radiomics based on early summation images (5-15 min p.i.), while no reliable information about the TERTp-mutation status was possible based on the standard summation images (20-40 min p.i.).

Previous studies have shown that patients with IDHwildtype TERTp-mutant glioblastoma have a significantly

Table 2 Coefficients of selected features in the TTP model

\begin{tabular}{lc}
\hline Features & Coefficients \\
\hline SmallDependenceLowGreyLevelEmphasis & 1.508 \\
Energy & 1.404 \\
SmallDependenceHighGreyLevelEmphasis & -1.283 \\
GreyLevelNonUniformityNormalized & -1.235 \\
LeastAxisLength & -1.219 \\
Busyness & -0.916 \\
ShortRunHighGreyLevelEmphasis & -0.699 \\
Maximum2DDiameterColumn & 0.654 \\
LowGreyLevelZoneEmphasis & -0.626 \\
LargeDependenceHighGreyLevelEmphasis & 0.606 \\
\hline
\end{tabular}

Intercept $\theta_{0}$ is 0.599 in the TTP model. Details of features were shown in Supplementary Information shorter progression free and overall survival compared to those with TERT-wildtype status. Therefore, TERTp-mutation status is now considered to be an important diagnostic and prognostic factor in primary glioblastomas and especially in patients with IDH-wildtype glioma [3, 5, 8, 9, 48]. TERTp-mutations indicate tumours that require aggressive and immediate treatments [3]. Hence, a preoperative tool for the prediction of a TERTp-mutation would be useful for early decision making and clinical management of patients with suspected glioma.

Several studies have analyzed the value of MRI based radiomics to predict the TERTp-mutation status in brain tumour patients [49-51]. Although these studies reported to achieve high accuracy values in the range of 79.88-93.80\%, only WHO grade II or/and III gliomas have been considered and a limited number of patients has been investigated [49-51]. Besides, Tian et al. established a multiparameter MRI based radiomics model for the prediction of the TERTp-mutation status in patients with high-grade glioma [52], but ignored that TERTp-mutations play different roles in different IDH phenotypes [48].

Compared with conventional MRI, amino acid PET has been shown to be more sensitive in the definition of brain tumour extent [39], and dynamic $\left[{ }^{18} \mathrm{~F}\right] \mathrm{FET}$ uptake parameters extracted from the TAC have shown to be an independent biomarker for prognosis [53, 54]. Several studies have reported the informative value of $\left[{ }^{18}\right.$ F]FET PET-based radiomics in personalized clinical decisions and individualized treatment selection [27-29, 55]. Lohmann et al. found textural feature analysis in combination with TBRs to better differentiate brain metastasis recurrence from radiation injury than TBRs alone, and $\left[{ }^{18}\right.$ F]FET PET radiomics achieved a higher accuracy than the best standard FET PET parameter $\left(\mathrm{TBR}_{\max }\right)$ to diagnose patients with pseudoprogression $[27,55]$. Haubold et al. utilized multiparametric $\left[{ }^{18} \mathrm{~F}\right] \mathrm{FET}$ PET/MRI and MR fingerprinting to decode and phenotype cerebral gliomas, which may serve as an alternative to invasive tissue characterization [28]. In addition, Carles et al. evaluated the prognostic value of $\left[{ }^{18} \mathrm{~F}\right]$ FET PET radiomics after re-irradiation, and found it could contribute to the selection of recurrent glioblastoma patients benefiting from re-irradiation [29]. However, all studies included radiomics based on standard static images (20-40 min p.i.) only and did not extract radiomic features derived from dynamic $\left[{ }^{18} \mathrm{~F}\right]$ FET PET as well as early summation images (5-15 min p.i.) even though two studies have shown the impact of dynamic parameters on radiomics $[32,56]$. Furthermore, no study has evaluated the potential to predict the TERTp-mutation status by $\left[{ }^{18}\right.$ F]FET PET radiomics so far.

This study included standard static images (20-40 min p.i.), early summation images (5-15 min p.i.), and dynamic $\left[{ }^{18} \mathrm{~F}\right] \mathrm{FET}$ PET images to develop the radiomic models. A total of 107 features were extracted from each 

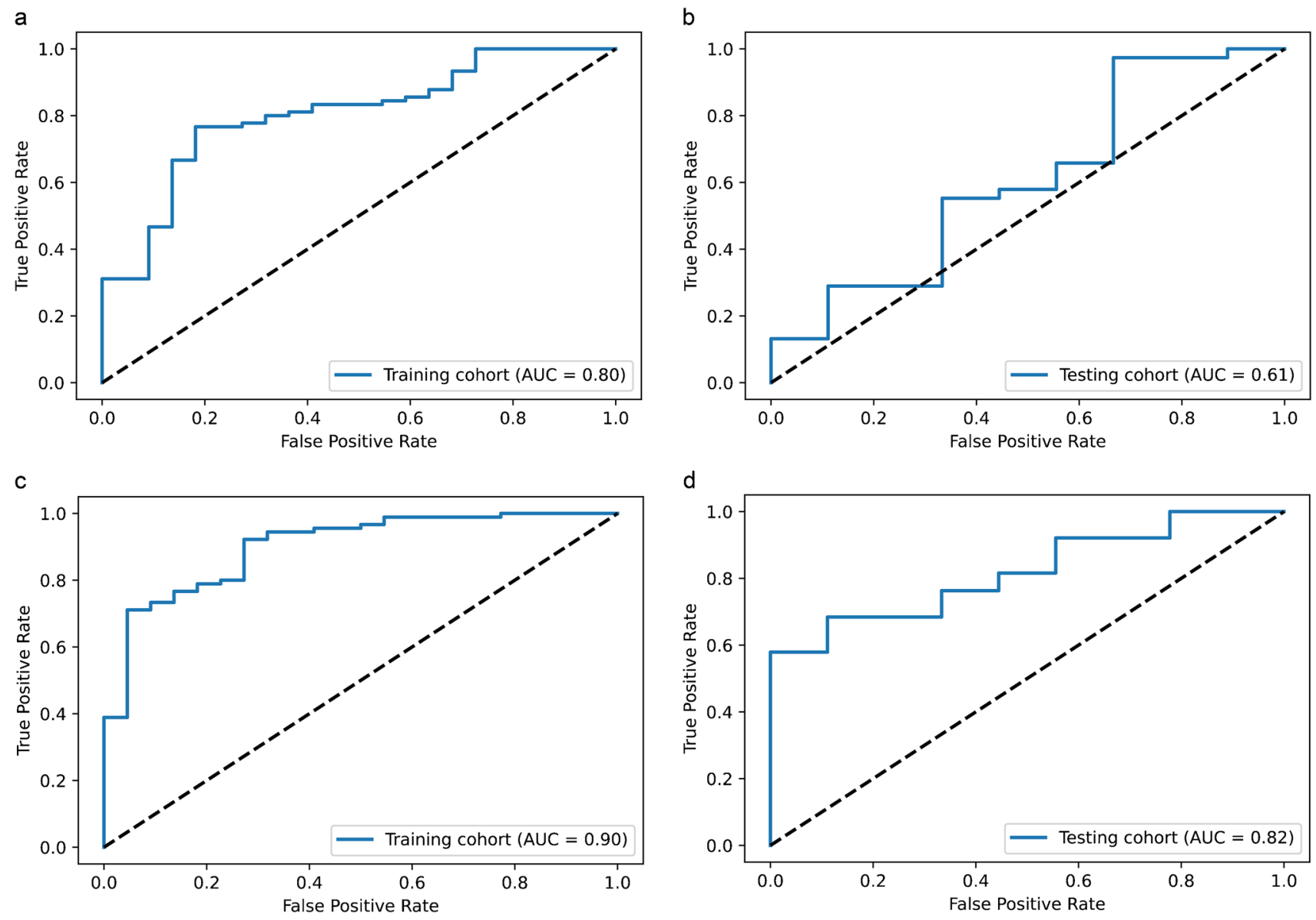

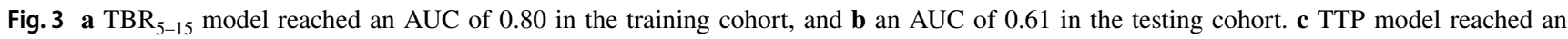
AUC of 0.90 in the training cohort, and $\mathbf{d}$ an AUC of 0.82 in the testing cohort. AUC area under the receiver operating characteristic curve

image. Our TTP model, built from ten dynamic $\left[{ }^{18} \mathrm{~F}\right]$ FET PET features selected by RFE, achieved the highest AUC of 0.82 in the independent testing cohort, indicating that the TERTp-mutation status can be predicted by using $\left[{ }^{18}\right.$ F]FET PET based radiomics. Notably, our former study did neither find an association between the TERTp-mutation status and traditional static $\left[{ }^{18} \mathrm{~F}\right]$
FET PET parameters $\left(\mathrm{TBR}_{\max }\right.$ and $\mathrm{TBR}_{\text {mean }}$ in static 20-40 min summation images) nor the standard dynamic parameter $\mathrm{TTP}_{\min }[24]$.

Interestingly, radiomics based on the standard $\mathrm{TBR}_{20-40}$ model showed a low performance for the prediction of the TERTp-mutation status, and even the $\mathrm{TBR}_{5-15}$ model, generated from nine early summation $\left[{ }^{18} \mathrm{~F}\right]$ FET PET features,

Table 3 Performance of each model

\begin{tabular}{|c|c|c|c|c|c|c|}
\hline & \multicolumn{2}{|l|}{$\mathrm{TBR}_{5-15}$} & \multicolumn{2}{|l|}{$\mathrm{TBR}_{20-40}$} & \multicolumn{2}{|l|}{ TTP } \\
\hline & Training cohort & Testing cohort & Training cohort & Testing cohort & Training cohort & Testing cohort \\
\hline AUC & 0.80 & 0.61 & 0.90 & 0.49 & 0.90 & 0.82 \\
\hline AUC 95\%CI & $(0.71-0.89)$ & $(0.42-0.80)$ & $(0.85-0.95)$ & $(0.30-0.69)$ & $(0.84-0.95)$ & $(0.71-0.92)$ \\
\hline Accuracy & $0.75 .0 \%$ & $66.0 \%$ & $83.0 \%$ & $66.0 \%$ & $78.6 \%$ & $83.0 \%$ \\
\hline Sensitivity & $73.3 \%$ & $73.7 \%$ & $81.1 \%$ & $73.7 \%$ & $77.8 \%$ & $92.1 \%$ \\
\hline Specificity & $81.8 \%$ & $33.3 \%$ & $90.9 \%$ & $33.3 \%$ & $81.8 \%$ & $44.4 \%$ \\
\hline PPV & $94.3 \%$ & $82.4 \%$ & $97.3 \%$ & $82.4 \%$ & $94.6 \%$ & $87.5 \%$ \\
\hline NPV & $42.9 \%$ & $23.1 \%$ & $54.1 \%$ & $23.1 \%$ & $47.4 \%$ & $57.1 \%$ \\
\hline
\end{tabular}

CI confidence interval 
had an accuracy of only $66 \%$ and an AUC of 0.61 in the testing cohort. With a high prediction accuracy of $83 \%$ in the TTP model, our study demonstrates that radiomic features extracted from dynamic PET data can achieve a higher performance level than models based on static PET data. Remarkably, the sensitivity of the TTP model reached $92.1 \%$ in the testing cohort, so that patients with aggressive TERTp-mutant glioma can be identified non-invasively with high probability [3]. With the generated multivariate LRbased formula, health practitioners will be able to calculate the patient individual risk probability of bearing a TERTpmutation before neurosurgical intervention. Our study shows that even sophisticated radiomic analysis of static $\left[{ }^{18} \mathrm{~F}\right] \mathrm{FET}$ PET imaging cannot replace dynamic acquisitions, at least with regard to the prediction of the TERTp-mutation status.

Traditional dynamic $\left[{ }^{18} \mathrm{~F}\right] \mathrm{FET}$ PET parameters such as the classification of the time-activity curve (increasing vs. decreasing or increasing vs. plateau vs. decreasing), the slope or the $\mathrm{TTP}_{\min }$ were most frequently calculated from a mean VOI-TAC of the tumour or from the hot-spot of the tumour with a $90 \%$ isocontour [10, 12, 19]. Considering the heterogeneity of gliomas, it may happen that the hotspot in standard summation images does not correspond to the most suspicious tumour aggressiveness when only considering $\mathrm{TTP}_{\min }$ and TAC and that, therefore, the most aggressive areas are inadvertently not evaluated. In contrast, we extracted the dynamic $\left[{ }^{18} \mathrm{~F}\right] \mathrm{FET}$ uptake information in every voxel within the tumour VOI and generated TTP images. This approach, which was first introduced by Kaiser et al. [40, 42], ensures that the dynamic information including the heterogeneity of uptake kinetics is extracted and that radiomics can be performed on the prognostically valuable dynamic data. The correlation between tumour heterogeneity and TERTp-mutation status can be considered in GreyLevelNonUniformityNormalized (GLNN) feature, which was used in the TTP model (see Table 2). GLNN belongs to Gray Level Dependence Matrix (GLDM), which is mathematically equal to first order-uniformity and is a measure of the homogeneity of the image array. A low value implies a greater heterogeneity, which was correlated with the TERTp-mutation, indicating that tumours with more heterogeneous TTP images are more likely to be classified as TERTp-mutant glioma.

Several limitations of this study should be discussed. First, the number of investigated patients is relatively small. However, it needs to be considered that we analyzed a very homogeneous group of patients with newly diagnosed and untreated IDH-wildtype diffuse astrocytic high-grade glioma. To exclude any influence by scanner type, all images in this study were derived from the same PET scanner, which limited the number of patients as well. In order to increase the number of patients, multi-centre validation studies are needed which, however, require phantom studies and harmonization of reconstruction parameters to make images from different PET scanners comparable. Another approach to directly harmonize features extracted from different devices may be to use the ComBat method [57]. In addition, our results are difficult to extrapolate to other centres, as the PET images analyzed in this study were acquired with our old PET scanner with fixed time frames, resulting in relatively long time frames (predominantly 5 and $10 \mathrm{~min}$ ) in the dynamic analysis which could not be changed afterwards, and were reconstructed using filtered back-projection, while most PET centres now use other reconstruction methods such as ordered subset expectation maximization (OSEM). Furthermore, radiomic features were only extracted from the $\left[{ }^{18} \mathrm{~F}\right]$ FET-positive tumour VOI to construct the model. Besides the tumour VOI, the remaining image (with normal seeming tissue) may still contain invisible but useful information. To analyze the entire images, deep learning methods will be necessary. Furthermore, our study focused on PET-based radiomics only. A combination with MRI may improve the performance of the prediction model and should be evaluated in future studies.

\section{Conclusion}

While conventional $\left[{ }^{18} \mathrm{~F}\right]$ FET PET parameters assessed by standard analyses have previously shown no association with the TERTp-mutation status, radiomic models can predict the TERTp-mutation status of IDH-wildtype diffuse astrocytic high-grade gliomas with high accuracy preoperatively. Notably, this is only the case for radiomics based on dynamic image data (TTP model) instead of standard summation images (20-40 min). Further external validation in multicentre studies with a larger number of patients is needed to evaluate the potential for clinical applications.

Supplementary Information The online version contains supplementary material available at https://doi.org/10.1007/s00259-021-05526-6.

Author contribution Conceptualization: Zhicong Li and Nathalie L. Albert; Data acquisition: Adrien Holzgreve, Viktoria C. Ruf, Bogdana Suchorska, Vera Wenter, Stefanie Quach, Jochen Herms and Marcus Unterrainer; Image processing: Lena Kaiser; Implementation and application of the software for generation of parametric maps and for radiomic feature extraction using Pyradiomics: Lena Kaiser; Feature selection and machine learning implementation and application: Zhicong Li; Formal analysis: Adrien Holzgreve; Writing —original draft preparation: Zhicong Li, Lena Kaiser and Nathalie L. Albert; Writing-review and editing: All; Funding acquisition, Zhicong Li and Nathalie L. Albert; Supervision: Peter Bartenstein, Jörg-Christian Tonn and Nathalie L. Albert. All authors have read and agreed to the published version of the manuscript.

Funding Open Access funding enabled and organized by Projekt DEAL. This work was supported by the Collaborative Research Centre SFB-824 of the Deutsche Forschungsgemeinschaft (DFG) and by the 
Else Kröner-Fresenius-Stiftung. The China Scholarship Council (CSC) funded this study for Zhicong Li.

Data availability The data that support the findings of this study are available on request from the corresponding author, N.L.A. The data are not publicly available due to the privacy of research participants.

Code availability The codes that support the findings of this study are available from the Z.L. and L.K., upon reasonable request.

\section{Declarations}

Ethics approval Ethical approval of the retrospective data analysis was given by the institutional review board of the LMU (\# 606-16) in accordance with the ICH Guideline for Good Clinical Practice (GCP) and the Declaration of Helsinki.

Consent to participate All patients have given written informed consent prior to the PET examination.

\section{Consent for publication Not applicable.}

Conflict of interest N.L.A. is a member of the Neuroimaging Committee of the EANM. All other authors declare that they have no relationships or interests that could have direct or potential influence or impart bias on the work.

Open Access This article is licensed under a Creative Commons Attribution 4.0 International License, which permits use, sharing, adaptation, distribution and reproduction in any medium or format, as long as you give appropriate credit to the original author(s) and the source, provide a link to the Creative Commons licence, and indicate if changes were made. The images or other third party material in this article are included in the article's Creative Commons licence, unless indicated otherwise in a credit line to the material. If material is not included in the article's Creative Commons licence and your intended use is not permitted by statutory regulation or exceeds the permitted use, you will need to obtain permission directly from the copyright holder. To view a copy of this licence, visit http://creativecommons.org/licenses/by/4.0/.

\section{References}

1. Arita H, Narita Y, Takami H, Fukushima S, Matsushita Y, Yoshida A, et al. TERT promoter mutations rather than methylation are the main mechanism for TERT upregulation in adult gliomas. Acta Neuropathol. 2013;126:939-41. https://doi.org/10.1007/ s00401-013-1203-9.

2. Killela PJ, Reitman ZJ, Jiao Y, Bettegowda C, Agrawal N, Diaz LA Jr, et al. TERT promoter mutations occur frequently in gliomas and a subset of tumors derived from cells with low rates of self-renewal. Proc Natl Acad Sci U S A. 2013;110:6021-6. https:// doi.org/10.1073/pnas.1303607110.

3. Simon M, Hosen I, Gousias K, Rachakonda S, Heidenreich B, Gessi M, et al. TERT promoter mutations: a novel independent prognostic factor in primary glioblastomas. Neuro Oncol. 2015;17:45-52. https://doi.org/10.1093/neuonc/nou158.

4. Eckel-Passow JE, Lachance DH, Molinaro AM, Walsh KM, Decker PA, Sicotte H, et al. Glioma groups based on $1 \mathrm{p} / 19 \mathrm{q}$, IDH, and TERT promoter mutations in tumors. N Engl J Med. 2015;372:2499-508. https://doi.org/10.1056/NEJMoa1407279.
5. Labussière M, Di Stefano AL, Gleize V, Boisselier B, Giry M, Mangesius S, et al. TERT promoter mutations in gliomas, genetic associations and clinico-pathological correlations. Br J Cancer. 2014;111:2024-32. https://doi.org/10.1038/bjc.2014.538.

6. Vinagre J, Almeida A, Pópulo H, Batista R, Lyra J, Pinto V, et al. Frequency of TERT promoter mutations in human cancers. Nat Commun. 2013;4:2185. https://doi.org/10.1038/ncomms3185.

7. Rushing EJ. WHO classification of tumors of the nervous system: preview of the upcoming 5th edition. memo - Magazine of European Medical Oncology. 2021. https://doi.org/10.1007/ s12254-021-00680-x.

8. Brat DJ, Aldape K, Colman H, Holland EC, Louis DN, Jenkins RB, et al. cIMPACT-NOW update 3: recommended diagnostic criteria for "Diffuse astrocytic glioma, IDH-wildtype, with molecular features of glioblastoma, WHO grade IV." Acta Neuropathol. 2018;136:805-10. https://doi.org/10.1007/ s00401-018-1913-0.

9. Louis DN, Wesseling P, Aldape K, Brat DJ, Capper D, Cree IA, et al. cIMPACT-NOW update 6: new entity and diagnostic principle recommendations of the cIMPACT-Utrecht meeting on future CNS tumor classification and grading. Brain Pathol. 2020;30:844 56. https://doi.org/10.1111/bpa.12832.

10. Jansen NL, Suchorska B, Wenter V, Schmid-Tannwald C, Todica A, Eigenbrod S, et al. Prognostic significance of dynamic $18 \mathrm{~F}$ FET PET in newly diagnosed astrocytic high-grade glioma. J Nucl Med. 2015;56:9-15. https://doi.org/10.2967/jnumed.114.144675.

11. Jansen NL, Suchorska B, Wenter V, Eigenbrod S, Schmid-Tannwald C, Zwergal A, et al. Dynamic 18F-FET PET in newly diagnosed astrocytic low-grade glioma identifies high-risk patients. J Nucl Med. 2014;55:198-203. https://doi.org/10.2967/jnumed.113. 122333.

12. Popperl G, Kreth FW, Mehrkens JH, Herms J, Seelos K, Koch W, et al. FET PET for the evaluation of untreated gliomas: correlation of FET uptake and uptake kinetics with tumour grading. Eur J Nucl Med Mol Imaging. 2007;34:1933-42. https://doi.org/10. 1007/s00259-007-0534-y.

13. Albert NL, Weller M, Suchorska B, Galldiks N, Soffietti R, Kim $\mathrm{MM}$, et al. Response assessment in neuro-oncology working group and European Association for Neuro-Oncology recommendations for the clinical use of PET imaging in gliomas. Neuro Oncol. 2016;18:1199-208. https://doi.org/10.1093/neuonc/now058.

14. Langen KJ, Watts C. Neuro-oncology: amino acid PET for brain tumours - ready for the clinic? Nat Rev Neurol. 2016;12:375-6. https://doi.org/10.1038/nrneurol.2016.80.

15. Galldiks N, Langen KJ, Albert NL, Chamberlain M, Soffietti R, Kim MM, et al. PET imaging in patients with brain metastasisreport of the RANO/PET group. Neuro Oncol. 2019;21:585-95. https://doi.org/10.1093/neuonc/noz003.

16. Law I, Albert NL, Arbizu J, Boellaard R, Drzezga A, Galldiks $\mathrm{N}$, et al. Joint EANM/EANO/RANO practice guidelines/SNMMI procedure standards for imaging of gliomas using PET with radiolabelled amino acids and [(18)F]FDG: version 1.0. Eur J Nucl Med Mol Imaging. 2019;46:540-57. https://doi.org/10.1007/ s00259-018-4207-9.

17. Albert NL, Winkelmann I, Suchorska B, Wenter V, Schmid-Tannwald C, Mille E, et al. Early static (18)F-FET-PET scans have a higher accuracy for glioma grading than the standard 20-40 min scans. Eur J Nucl Med Mol Imaging. 2016;43:1105-14. https:// doi.org/10.1007/s00259-015-3276-2.

18. Kunz M, Albert NL, Unterrainer M, la Fougere C, Egensperger $\mathrm{R}$, Schüller U, et al. Dynamic 18F-FET PET is a powerful imaging biomarker in gadolinium-negative gliomas. Neuro Oncol. 2019;21:274-84. https://doi.org/10.1093/neuonc/noy098.

19. Jansen NL, Graute V, Armbruster L, Suchorska B, Lutz J, Eigenbrod S, et al. MRI-suspected low-grade glioma: is there a need 
to perform dynamic FET PET? Eur J Nucl Med Mol Imaging. 2012;39:1021-9. https://doi.org/10.1007/s00259-012-2109-9.

20. Jansen NL, Suchorska B, Wenter V, Eigenbrod S, Schmid-Tannwald C, Zwergal A, et al. Dynamic 18F-FET PET in newly diagnosed astrocytic low-grade glioma identifies high-risk patients. J Nucl Med. 2014;55:198-203. https://doi.org/10.2967/jnumed.113. 122333.

21. Fleischmann DF, Unterrainer M, Bartenstein P, Belka C, Albert NL, Niyazi M. 18F-FET PET prior to recurrent high-grade glioma re-irradiation-additional prognostic value of dynamic time-topeak analysis and early static summation images? J Neurooncol. 2017;132:277-86. https://doi.org/10.1007/s11060-016-2366-8.

22. Vettermann F, Suchorska B, Unterrainer M, Nelwan D, Forbrig R, Ruf V, et al. Non-invasive prediction of IDH-wildtype genotype in gliomas using dynamic 18F-FET PET. Eur J Nucl Med Mol Imaging. 2019;46:2581-9. https://doi.org/10.1007/ s00259-019-04477-3.

23. Suchorska B, Giese A, Biczok A, Unterrainer M, Weller M, Drexler M, et al. Identification of time-to-peak on dynamic 18F-FETPET as a prognostic marker specifically in IDH1/2 mutant diffuse astrocytoma. Neuro Oncol. 2018;20:279-88. https://doi.org/10. 1093/neuonc/nox 153 .

24. Unterrainer M, Ruf V, von Rohr K, Suchorska B, Mittlmeier LM, Beyer L, et al. TERT-promoter mutational status in glioblastoma-is there an association with amino acid uptake on dynamic (18)F-FET PET? Front Oncol. 2021;11:645316. https://doi.org/ 10.3389/fonc.2021.645316.

25. Gillies RJ, Kinahan PE, Hricak H. Radiomics: images are more than pictures, they are data. Radiology. 2016;278:563-77. https:// doi.org/10.1148/radiol.2015151169.

26. Lambin P, Rios-Velazquez E, Leijenaar R, Carvalho S, van Stiphout RG, Granton P, et al. Radiomics: extracting more information from medical images using advanced feature analysis. Eur J Cancer. 2012;48:441-6. https://doi.org/10.1016/j.ejca.2011.11. 036.

27. Lohmann P, Elahmadawy MA, Gutsche R, Werner JM, Bauer EK, Ceccon G, et al. FET PET radiomics for differentiating pseudoprogression from early tumor progression in glioma patients post-chemoradiation. Cancers (Basel). 2020;12. https://doi.org/ $10.3390 /$ cancers 12123835 .

28. Haubold J, Demircioglu A, Gratz M, Glas M, Wrede K, Sure U, et al. Non-invasive tumor decoding and phenotyping of cerebral gliomas utilizing multiparametric (18)F-FET PET-MRI and MR Fingerprinting. Eur J Nucl Med Mol Imaging. 2020;47:1435-45. https://doi.org/10.1007/s00259-019-04602-2.

29. Carles M, Popp I, Starke MM, Mix M, Urbach H, Schimek-Jasch $\mathrm{T}$, et al. FET-PET radiomics in recurrent glioblastoma: prognostic value for outcome after re-irradiation? Radiat Oncol. 2021;16:46. https://doi.org/10.1186/s13014-020-01744-8.

30. Qian J, Herman MG, Brinkmann DH, Laack NN, Kemp BJ, Hunt $\mathrm{CH}$, et al. Prediction of MGMT status for glioblastoma patients using radiomics feature extraction from (18)F-DOPA-PET imaging. Int J Radiat Oncol Biol Phys. 2020;108:1339-46. https://doi. org/10.1016/j.ijrobp.2020.06.073.

31. Lohmann P, Meißner AK, Kocher M, Bauer EK, Fink GR, et al. Feature-based PET/MRI radiomics in patients with brain tumors. Neurooncol Adv. 2020;2:iv15-21. https://doi.org/10.1093/noajnl/ vdaa118.

32. Zaragori T, Oster J, Roch V, Hossu G, Chawki MB, Grignon R, et al. (18)F-FDOPA PET for the non-invasive prediction of glioma molecular parameters: a radiomics study. J Nucl Med. 2021. https://doi.org/10.2967/jnumed.120.261545.

33. Louis DN, Perry A, Reifenberger G, von Deimling A, FigarellaBranger D, Cavenee WK, et al. The 2016 World Health Organization Classification of Tumors of the Central Nervous System: a summary. Acta Neuropathol. 2016;131:803-20. https://doi.org/ 10.1007/s00401-016-1545-1.

34. Louis DN, Ohgaki H, Wiestler OD, Cavenee WK, Burger PC, Jouvet A, et al. The 2007 WHO classification of tumours of the central nervous system. Acta Neuropathol. 2007;114:97-109. https://doi.org/10.1007/s00401-007-0243-4.

35. Thon N, Eigenbrod S, Kreth S, Lutz J, Tonn JC, Kretzschmar H, et al. IDH1 mutations in grade II astrocytomas are associated with unfavorable progression-free survival and prolonged postrecurrence survival. Cancer. 2012;118:452-60. https://doi.org/10.1002/ cncr.26298.

36. Biczok A, Kraus T, Suchorska B, Terpolilli NA, Thorsteinsdottir J, Giese A, et al. TERT promoter mutation is associated with worse prognosis in WHO grade II and III meningiomas. J Neurooncol. 2018;139:671-8. https://doi.org/10.1007/s11060-018-2912-7.

37. Langen KJ, Bartenstein P, Boecker H, Brust P, Coenen HH, Drzezga A, et al. German guidelines for brain tumour imaging by PET and SPECT using labelled amino acids. Nuklearmedizin. 2011;50:167-73. https://doi.org/10.3413/nuk-2011041.

38. Unterrainer M, Vettermann F, Brendel M, Holzgreve A, Lifschitz $\mathrm{M}$, Zahringer M, et al. Towards standardization of 18F-FET PET imaging: do we need a consistent method of background activity assessment? EJNMMI Res. 2017;7:48.

39. Pauleit D, Floeth F, Hamacher K, Riemenschneider MJ, Reifenberger G, Muller HW, et al. O-(2-[18F]fluoroethyl)-L-tyrosine PET combined with MRI improves the diagnostic assessment of cerebral gliomas. Brain. 2005;128:678-87. https://doi.org/10. 1093/brain/awh399.

40. Vomacka L, Unterrainer M, Holzgreve A, Mille E, Gosewisch A, Brosch J, et al. Voxel-wise analysis of dynamic 18F-FET PET: a novel approach for non-invasive glioma characterisation. EJNMMI Res. 2018;8:91. https://doi.org/10.1186/s13550-018-0444-y.

41. Van Griethuysen JJM, Fedorov A, Parmar C, Hosny A, Aucoin N, Narayan V, et al. Computational radiomics system to decode the radiographic phenotype. Can Res. 2017;77:e104-7. https://doi. org/10.1158/0008-5472.can-17-0339.

42. Kaiser LGM, Ahmadi SA, Unterrainer M, Holzgreve A, Mille E, Gosewisch A, Brosch J, Suchorska B, Navab N, Tonn JC, Ziegler S, Bartenstein P, Albert NL, Böning G. Annual Congress of the European Association of Nuclear Medicine October 12-16, 2019 Barcelona, Spain. Eur J Nucl Med Mol Imaging. 2019;46:1-952. https://doi.org/10.1007/s00259-019-04486-2.

43. Zwanenburg A, Leger $\mathrm{S}$, Vallières $\mathrm{M}$, Löck S. Image biomarker standardisation initiative. arXiv preprint arXiv:161207003. 2016.

44. Leijenaar RT, Nalbantov G, Carvalho S, van Elmpt WJ, Troost EG, Boellaard R, et al. The effect of SUV discretization in quantitative FDG-PET Radiomics: the need for standardized methodology in tumor texture analysis. Sci Rep. 2015;5:11075. https://doi. org/10.1038/srep11075.

45. Granitto PM, Furlanello C, Biasioli F, Gasperi F. Recursive feature elimination with random forest for PTR-MS analysis of agroindustrial products. Chemometr Intell Lab. 2006;83:83-90. https://doi.org/10.1016/j.chemolab.2006.01.007.

46. Tomz M, King G, Zeng L. ReLogit: rare events logistic regression. 2003. 2003;8:27. https://doi.org/10.18637/jss.v008.i02.

47. Makhoul J. Linear prediction: a tutorial review. Proc IEEE. 1975;63:561-80. https://doi.org/10.1109/PROC.1975.9792.

48. Vuong HG, Altibi AMA, Duong UNP, Ngo HTT, Pham TQ, Chan AK, et al. TERT promoter mutation and its interaction with IDH mutations in glioma: combined TERT promoter and IDH mutations stratifies lower-grade glioma into distinct survival subgroups-A meta-analysis of aggregate data. Crit Rev Oncol Hematol. 2017;120:1-9. https://doi.org/10.1016/j.critrevonc.2017.09. 013. 
49. Jiang C, Kong Z, Zhang Y, Liu S, Liu Z, Chen W, et al. Conventional magnetic resonance imaging-based radiomic signature predicts telomerase reverse transcriptase promoter mutation status in grade II and III gliomas. Neuroradiology. 2020;62:803-13. https:// doi.org/10.1007/s00234-020-02392-1.

50. Park CJ, Han K, Kim H, Ahn SS, Choi D, Park YW, et al. MRI features may predict molecular features of glioblastoma in isocitrate dehydrogenase wild-type lower-grade gliomas. AJNR Am J Neuroradiol. 2021;42:448-56. https://doi.org/10.3174/ajnr.A6983.

51. Fang S, Fan Z, Sun Z, Li Y, Liu X, Liang Y, et al. Radiomics features predict telomerase reverse transcriptase promoter mutations in world health organization grade II gliomas via a machinelearning approach. Front Oncol. 2020;10:606741. https://doi.org/ 10.3389/fonc. 2020.606741

52. Tian $\mathrm{H}, \mathrm{Wu} \mathrm{H}, \mathrm{Wu} \mathrm{G}, \mathrm{Xu} \mathrm{G}$. Noninvasive prediction of TERT promoter mutations in high-grade glioma by radiomics analysis based on multiparameter MRI. Biomed Res Int. 2020;2020:3872314. https://doi.org/10.1155/2020/3872314.

53. Kunz M, Thon N, Eigenbrod S, Hartmann C, Egensperger R, Herms J, et al. Hot spots in dynamic (18)FET-PET delineate malignant tumor parts within suspected WHO grade II gliomas. Neuro Oncol. 2011;13:307-16. https://doi.org/10.1093/neuonc/ noq196.
54. Bauer EK, Stoffels G, Blau T, Reifenberger G, Felsberg J, Werner $\mathrm{JM}$, et al. Prediction of survival in patients with IDH-wildtype astrocytic gliomas using dynamic O-(2-[(18)F]-fluoroethyl)-Ltyrosine PET. Eur J Nucl Med Mol Imaging. 2020;47:1486-95. https://doi.org/10.1007/s00259-020-04695-0.

55. Lohmann P, Stoffels G, Ceccon G, Rapp M, Sabel M, Filss $\mathrm{CP}$, et al. Radiation injury vs. recurrent brain metastasis: combining textural feature radiomics analysis and standard parameters may increase (18)F-FET PET accuracy without dynamic scans. Eur Radiol. 2017;27:2916-27. https://doi.org/10.1007/ s00330-016-4638-2.

56. Lohmann P, Lerche C, Bauer EK, Steger J, Stoffels G, Blau $\mathrm{T}$, et al. Predicting IDH genotype in gliomas using FET PET radiomics. Sci Rep. 2018;8:13328. https://doi.org/10.1038/ s41598-018-31806-7.

57. Orlhac F, Boughdad S, Philippe C, Stalla-Bourdillon H, Nioche $\mathrm{C}$, Champion L, et al. A postreconstruction harmonization method for multicenter radiomic studies in PET. J Nucl Med. 2018;59:1321-8. https://doi.org/10.2967/jnumed.117.199935.

Publisher's note Springer Nature remains neutral with regard to jurisdictional claims in published maps and institutional affiliations. 